International Journal of Pure and Applied Mathematics

Volume 117 No. 1 2017, 11-18

ISSN: 1311-8080 (printed version); ISSN: 1314-3395 (on-line version)

url: http://www.ijpam.eu

doi: $10.12732 /$ ijpam.v117i1.2

\title{
INTEGRAL OPERATOR IN AMALGAM
}

\author{
Suket Kumar \\ Department of Mathematics \\ NIT Hamirpur \\ H.P., 177005, INDIA
}

Abstract: Boundedness of the sum of two Hardy-type operators with not necessarily nonnegative coefficients has been discussed between amalgams $\ell^{q}\left(X_{u}\right)-\ell^{b}\left(L^{r}, v\right)$ for the case $1<r, q, b<\infty$ where $X_{u}$ is weighted Banach function space.

AMS Subject Classification: 26D10, 26D15

Key Words: boundedness, amalgams, Banach function space, Hardy-type operator

\section{Introduction}

Consider the operator

$$
(S f)(x)=\phi_{1}(x) \int_{-\infty}^{x} \psi_{1}(t) f(t) d t+\phi_{2}(x) \int_{x}^{\infty} \psi_{2}(t) f(t) d t
$$

where $\phi_{i}, \psi_{i} ; i=1,2$ are non-zero measurable functions not necessarily nonnegative and $f$ is positive measurable function. Boundedness of $S$ between weighted Lebesgue spaces in one dimension has been considered in [8 (Remark 2.4), 15], between weighted Lebesgue spaces in two dimension in [9], between suitable weighted amalgam spaces $\ell^{q}\left(L^{p}, u\right)-\ell^{\bar{q}}\left(L^{\bar{p}}, v\right)$ in [7] where $u, v$ are weight function, between Banach function space (BFS) in [10]. Boundedness conditions for the sum of two Hardy-type operators gives better value of best constant for Hardy inequality than the same for single Hardy-type operator.

$\begin{array}{ll}\text { Received: } & 2016-11-09 \\ \text { Revised: } & 2017-07-05\end{array}$

Published: November 25, 2017

(C) 2017 Academic Publications, Ltd. url: www.acadpubl.eu 
Motivated by this, in this paper, we have considered the boundedness of $S$ between $\ell^{q}\left(X_{u}\right)$ and $\ell^{b}\left(L^{r}, v\right)$ for certain ranges of indices, where $X_{u}$ is weighted BFS. Boundedness of the Hardy operator $(T f)(x)=\int_{-\infty}^{x} f(t) d t$ and it's adjoint operator $\left(T^{*} f\right)(x)=\int_{x}^{\infty} f(t) d t$ between $\ell^{q}\left(X_{u}\right)$ and $\ell^{b}\left(L^{r}, v\right)$ has been considered in [6], while $\ell^{q}\left(L^{p}, u\right)-\ell^{\bar{q}}\left(L^{\bar{p}}, v\right)$ boundedness of $T$ and $T^{*}$ has been proved in [3]. The notion of BFS was introduced by Luxemburg [12]. For detailed studies of BFS we used to refer [1]. Amalgams were introduced by Wiener $[13,14]$. A good treatment of such spaces is available in $[4,5]$. We use to refer [8] for the study of Hardy inequality.

Throughout the paper, the symbol $u$ and $v$ denotes weight function, that is, measurable functions, positive almost everywhere in the interval $(-\infty, \infty)$, $u_{n}$ and $v_{n}$ denotes sequence weight, $\chi_{n}$ denotes characteristic function over $[n, n+1]$ and $\chi_{[\alpha, \beta]}$ denotes characteristic function over $[\alpha, \beta], p^{\prime}=p /(p-1)$ denotes conjugate to $p$ and the same is true for other indices. For a BFS $X, X^{\prime}$ denotes it's adjoint space. $\mathbb{Z}$ represents the set of integers. We conclude this section by giving some results which will be further used as follows: below:

$\ell^{p}\left(v_{n}\right)-\ell^{q}\left(u_{n}\right)$ boundedness of the operator $H^{*}\left(a_{n}\right)=\sum_{k=n}^{\infty} a_{k}$, is stated

Proposition 1.1. Let $1<p, q<\infty, u_{n} \geq 0, v_{n}>0, n \in \mathbb{Z}$ and $\frac{1}{r}=\frac{1}{q}-\frac{1}{p}$. Then there exists a constant $C>0$ such that

$$
\left(\sum_{n \in \mathbb{Z}}\left(\sum_{k=n}^{\infty} a_{k}\right)^{q} u_{n}\right)^{1 / q} \leq C\left(\sum_{n \in \mathbb{Z}} a_{n}^{p} v_{n}\right)^{1 / p}
$$

holds for all non-negative sequence $\left\{a_{k}\right\} \in \ell^{q}\left(v_{n}\right)$, if and only if

(a) for $1<p \leq q<\infty$

$$
\sup _{m \in \mathbb{Z}}\left(\sum_{n=-\infty}^{m} u_{n}\right)^{1 / q}\left(\sum_{n=m}^{\infty} v_{n}^{1-p^{\prime}}\right)^{1 / p^{\prime}}<\infty,
$$

(b) for $1<q<b<\infty$

$$
\left\{\sum_{m \in \mathbb{Z}}\left(\sum_{n=-\infty}^{m} u_{k}\right)^{r / q}\left(\sum_{n=m}^{\infty} v_{n}^{1-p^{\prime}}\right)^{r / q^{\prime}} u_{m}\right\}^{1 / r}<\infty .
$$

Boundedness of the operator $\left(H_{1}^{*} f\right)(x)=\psi(x) \int_{x}^{\infty} \phi(t) f(t) d t$, between BFS $\mathrm{X}$ and $\mathrm{Y}$ where $\phi, \psi$ are weight function is stated below: 
Proposition 1.2. Let $\mathrm{X}, \mathrm{Y}$ are BFS satisfying the $\ell$-condition [2]. Then the inequality $\left\|H_{1}^{*} f\right\|_{Y} \leq C\|f\|_{X}$ holds for measurable function $f$ and a constant $\mathrm{C}$ if and only if $\sup _{0<t<\infty}\left\|\psi \chi_{[0, t]}\right\|_{Y}\left\|\phi \chi_{[t, \infty]}\right\|_{X^{\prime}}<\infty$.

The following result states the adjoint case of [6, Theorem 2]:

Proposition 1.3. Let $1<b, q, r<\infty, u, v$ are weight functions and $X_{u}$ is a weighted BFS. Suppose $X_{u}$ and the Lebesgue space $L^{r}$ satisfy $\ell$-condition. Then the inequality

$$
\left\|T^{*} f\right\|_{\ell q\left(X_{u}\right)} \leq C\|f\|_{r, v, b}
$$

holds for all $f \in \ell^{b}\left(L^{r}, u\right)$ if and only if for $b \leq q$

$$
\begin{aligned}
& A_{1}=\sup _{m \in \mathbb{Z}} \sup _{m<t<m+1}\left\|u \chi_{[m, t]}\right\|_{X}\left(\int_{t}^{m+1} v^{1-b^{\prime}}\right)^{1 / b^{\prime}}<\infty \\
& A_{2}=\sup _{m \in \mathbb{Z}}\left(\sum_{n=-\infty}^{m}\left\|u \chi_{n}\right\|_{X}^{q}\right)^{1 / q}\left(\sum_{n=m}^{\infty}\left(\int_{n-1}^{n} v^{1-r^{\prime}}\right)^{\frac{b^{\prime}}{r^{\prime}}}\right)^{1 / b^{\prime}}<\infty .
\end{aligned}
$$

\section{Boundedness of $S$ between Amalgam}

In this section we will consider $\ell^{q}\left(X_{u}\right)-\ell^{b}\left(L^{r}, v\right)$ boundedness of $\mathrm{S}$ for the case of $1<r, q, b<\infty$. First consider the following notations:

$$
\begin{aligned}
& C_{1}=\sup _{m \in \mathbb{Z}} \sup _{m<t<m+1}\left\|u \phi_{1} \chi_{[t, m+1]}\right\|_{X}\left(\int_{m}^{t}\left(v\left|\psi_{1}\right|^{-r}\right)^{1-r^{\prime}}\right)^{1 / r^{\prime}} \\
& C_{2}=\sup _{m \in \mathbb{Z}} \sup _{m<t<m+1}\left\|u \phi_{2} \chi_{[m, t]}\right\|_{X}\left(\int_{t}^{m+1}\left(v\left|\psi_{2}\right|^{-r}\right)^{1-r^{\prime}}\right)^{1 / r^{\prime}} \\
& C_{3}=\sup _{m \in \mathbb{Z}}\left(\sum_{n=m}^{\infty}\left\|u \phi_{1} \chi_{n}\right\|_{X}^{q}\right)^{1 / q}\left(\sum_{n=-\infty}^{m}\left(\int_{n-1}^{n}\left(v\left|\psi_{1}\right|^{-r}\right)^{1-r^{\prime}}\right)^{\frac{b^{\prime}}{r^{\prime}}}\right)^{1 / b^{\prime}} \\
& C_{4}=\sup _{m \in \mathbb{Z}}\left(\sum_{n=-\infty}^{m}\left\|u \phi_{2} \chi_{n}\right\|_{X}^{q}\right)^{1 / q}\left(\sum_{n=m}^{\infty}\left(\int_{n-1}^{n}\left(v\left|\psi_{2}\right|^{-r}\right)^{1-r^{\prime}}\right)^{\frac{b^{\prime}}{r^{\prime}}}\right)^{1 / b^{\prime}}
\end{aligned}
$$

where $\frac{1}{s}=\frac{1}{q}-\frac{1}{b}$. 
Theorem 2.1. Let $1<r, q, b<\infty, u, v$ are weight functions, $X_{u}$ is weighted BFS. Suppose $X_{u}$ and $L^{r}$ satisfy $\ell$-condition. Then there exist a constant $C>0$ for which the inequality

$$
\|S f\|_{\ell^{q}\left(X_{u}\right)} \leq C\|f\|_{r, v, b}
$$

holds for all $f \in \ell^{b}\left(L^{r}, v\right)$ if and only if for $b \leq q, \max \left(C_{1}, C_{2}, C_{3}, C_{4}\right)<\infty$.

Proof. Denote

$$
\left(S_{1} f\right)(x)=\phi_{1}(x) \int_{-\infty}^{x} \psi_{1}(t) f(t) d t ; \quad\left(S_{2} f\right)(x)=\phi_{2}(x) \int_{x}^{\infty} \psi_{2}(t) f(t) d t .
$$

Then $S=S_{1}+S_{2}$, consequently,

$$
\|S f\|_{\ell^{q}\left(X_{u}\right)} \leq\left\|S_{1} f\right\|_{\ell^{q}\left(X_{u}\right)}+\left\|S_{2} f\right\|_{\ell^{q}\left(X_{u}\right)} .
$$

Sufficiency. By an application of [6, Theorem 2(a)], the following can be proved.

Lemma A. Suppose $1<r, q, b<\infty, u, v$ are weight functions, $X_{u}$ is weighted BFS. Suppose $X_{u}$ and $L^{r}$ satisfy $\ell$-condition. Then there exist a constant $C>0$ for which the inequality

$$
\left\|S_{1} f\right\|_{\ell^{q}\left(X_{u}\right)} \leq C\|f\|_{r, v, b}
$$

holds for all $f \in \ell^{b}\left(L^{r}, v\right)$ if and only if for $b \leq q, \max \left(C_{1}, C_{3}\right)<\infty$.

In [6], Lemma A has been proved for $\phi_{1} \equiv \psi_{1} \equiv 1$ and $f \geq 0$. The proof of Lemma $\mathrm{A}$ is analogous to that proof. We omit the detail. When $X_{u}$ is weighted Lebesgue space, $\phi_{1} \equiv \psi_{1} \equiv 1$ and $f \geq 0$, Lemma $\mathrm{A}$ has been proved in [3].

The following can be proved from Proposition 1.3, by applying suitable substitutions:

Lemma B. Suppose $1<r, q, b<\infty, u, v$ are weight functions, $X_{u}$ is weighted BFS. Suppose $X_{u}$ and $L^{r}$ satisfy $\ell$-condition. Then there exist a constant $C>0$ for which the inequality

$$
\left\|S_{2} f\right\|_{\ell^{q}\left(X_{u}\right)} \leq C\|f\|_{r, v, b}
$$

holds for all $f \in \ell^{b}\left(L^{r}, v\right)$ if and only if for $b \leq q, \max \left(C_{2}, C_{4}\right)<\infty$.

Sufficiency now follows from Lemma A, Lemma B and the inequality (2.2).

Necessity. for non-negative functions, $\phi_{i}, \psi_{i}$. Suppose that the inequality (2.1) holds and that $f \geq 0$. Then

$$
\left\|S_{i} f\right\|_{\ell^{q}\left(X_{u}\right)} \leq\|S f\|_{\ell^{q}\left(X_{u}\right)} ; \quad i=1,2
$$


and consequently (2.3) and (2.4) holds. This implies that $\max \left(C_{1}, C_{3}\right)<\infty$ (for $b \leq q, i=1$ ), $\max \left(C_{2}, C_{4}\right)<\infty$ (for $b \leq q, i=2$ ). Necessity is now proved.

Necessity for general functions $\phi_{i}, \psi_{i}$. Suppose again that the inequality (2.1) holds. Define, for $\varepsilon>0$ and a measurable function $g>0$, a new weight function $v_{\varepsilon}$ as

$$
v_{\varepsilon}(x)=\max \left\{v(x), \frac{\left|\psi_{1}(x)\right|^{r}}{(g(x))^{r}} \varepsilon\right\}
$$

Since $v \leq v_{\varepsilon}$, we get $\|f\|_{r, v, b} \leq\|f\|_{r, v_{\varepsilon}, b}$, consequently the inequality (2.1) is equivalent to

$$
\|S f\|_{\ell^{q}\left(X_{u}\right)} \leq C\|f\|_{r, v_{\varepsilon}, b}
$$

Define

$$
f(x)=g(x)\left|\psi_{1}(x)\right|^{-1} \operatorname{sgn}\left(\psi_{1}(x)\right) \chi_{[\alpha, \beta]}(x)
$$

for $m \in \mathbb{Z}$ be fixed and $m<\alpha<\beta<m+1$. For $v_{\varepsilon}$ and $f$ defined above

$$
\begin{aligned}
\|f\|_{r, v_{\varepsilon}, b} & =\left(\int_{\alpha}^{\beta} g^{r}\left|\psi_{1}\right|^{-r} v_{\varepsilon}\right)^{1 / r} \leq\left(\int_{m}^{m+1} g^{r}\left|\psi_{1}\right|^{-r} v_{\varepsilon}\right)^{1 / r} \\
& \leq\left(\int_{m}^{m+1} g^{r}\left|\psi_{1}\right|^{-r}\left|\psi_{1}\right|^{r} g^{-r} \varepsilon\right)^{1 / r}=(\varepsilon)^{1 / r}<\infty
\end{aligned}
$$

and

$$
\begin{aligned}
\|S f\|_{\ell^{q}\left(X_{u}\right)} & =\left(\sum_{n \in \mathbb{Z}}\left\|S f . u \chi_{n}\right\|_{X}^{q}\right)^{1 / q}=\left\||S f| u \cdot \chi_{m}\right\|_{X} \geq\left\||S f| u \chi_{[\beta, m+1]}\right\|_{X} \\
& =\left\|\left|\phi_{1} \int_{-\infty}^{x} \psi_{1} f\right| u \chi_{[\beta, m+1]}\right\|_{X} \geq\left\|\left|\int_{m}^{x} g\right|\left(u\left|\phi_{1}\right|\right) \chi_{[\beta, m+1]}\right\|_{X}
\end{aligned}
$$

since $\int_{x}^{\infty} \psi_{2} f=0$ when $x>\beta$ of $f$ defined as (2.7). Consequently, the inequality (2.6) yields

$$
\left\|\left|\int_{m}^{x} g\right| \cdot\left(u\left|\phi_{1}\right|\right) \chi_{[\beta, m+1]} \mid\right\|_{X}\left(\int_{m}^{m+1} g^{r}\left|\psi_{1}\right|^{-r} v_{\varepsilon}\right)^{-1 / r} \leq C .
$$

Since $\mathrm{C}$ is independent of $\beta$ and $\varepsilon$, we have when $\beta \rightarrow m$ and $\varepsilon \rightarrow 0$ (via a subsequence)

$$
\left\|\left|\int_{m}^{x} g\right|\left(u\left|\phi_{1}\right|\right) \chi_{[m, m+1]}\right\|_{X} \leq C\left(\int_{m}^{m+1} g^{r}\left|\psi_{1}\right|^{-r} v\right)^{1 / r} .
$$


By an application of [11, Theorem 4] (also see [2]), this holds implies $C_{1}<\infty$. Next, we replace $v_{\varepsilon}$ and $f$ defined as in (2.5) and (2.7) by, respectively,

$$
v_{\varepsilon}(x)=\max \left\{v(x), \frac{\left|\psi_{2}(x)\right|^{r}}{(g(x))^{r}} \varepsilon\right\}
$$

and

$$
f(x)=g(x)\left|\psi_{2}(x)\right|^{-1} \operatorname{sgn}\left(\psi_{2}(x)\right) \chi_{[\alpha, \beta]}(x)
$$

where $m \in \mathbb{Z}$ is fixed and $m<\alpha<\beta<m+1$. The necessity of $C_{2}<\infty$ can be achieved by making parallel arguments in the inequality (2.6) for this new $v_{\varepsilon}$ and $f$ and using Proposition 1.2. For $\varepsilon>0$, we define

$$
\tilde{v}_{\varepsilon}(x)=\max \left\{v(x),\left|\psi_{1}(x)\right|^{r} \varepsilon\right\} .
$$

For the similar reason discussed as earlier, the inequality (2.1) is equivalent to

$$
\|S f\|_{\ell^{q}\left(X_{u}\right)} \leq C\|f\|_{r, \tilde{v}_{\varepsilon}, b} .
$$

For a non-negative sequence $\left\{a_{n}\right\} \in \ell^{b}$ and fixed $\alpha \in \mathbb{Z}$, define

$$
\tilde{f}(x)=\left\{\begin{array}{cc}
\sum_{n \in \mathbb{Z}} a_{n}\left(\frac{\tilde{v}_{\varepsilon}(x)}{\left|\psi_{1}(x)\right|}\right)^{1-r^{\prime}} \operatorname{sgn}\left(\psi_{1}(x)\right) \chi_{[n, n+1]}(x), & x \leq \alpha \\
0, & x>\alpha .
\end{array}\right.
$$

For $f$ defined as above

$$
\|\tilde{f}\|_{r, \tilde{v}_{\varepsilon}, b}=\left\{\sum_{n=-\infty}^{\alpha} A_{n}^{b} V_{n}\right\}^{1 / b} \leq\left\{\sum_{n \in \mathbb{Z}} A_{n}^{b} V_{n}\right\}^{1 / b}
$$

where

$$
A_{n}=a_{n-1} \int_{n-1}^{n}\left(\tilde{v}_{\varepsilon}\left|\psi_{1}\right|^{-r}\right)^{1-r^{\prime}} \text { and } V_{n}=\left(\int_{n-1}^{n}\left(\tilde{v}_{\varepsilon}\left|\psi_{1}\right|^{-r}\right)^{1-r^{\prime}}\right)^{-b / r^{\prime}} .
$$

Since $f$ defined as (2.10) vanishes for $x>\alpha$, we have for $\alpha<x<\alpha+1$

$$
(S \tilde{f})(x)=\phi_{1}(x)\left\{\sum_{n=-\infty}^{\alpha} \int_{n-1}^{n} \psi_{1}(t) \tilde{f}(t) d t\right\}=\phi_{1}(x) \sum_{n=-\infty}^{\alpha} A_{n}
$$

which implies

$$
\|S \tilde{f}\|_{X_{u}}=\left(\sum_{n=-\infty}^{\alpha} A_{n}\right)\left\|u \phi_{1} \chi_{\alpha}\right\|_{X}
$$


Consequently,

$$
\|S \tilde{f}\|_{\ell^{q}\left(X_{u}\right)}=\left\{\sum_{\alpha \in \mathbb{Z}}\left(\sum_{n=-\infty}^{\alpha} A_{n}\right)^{q}\left\|u \phi_{1} \chi_{\alpha}\right\|_{X}^{q}\right\}^{1 / q}
$$

the inequality (2.9) yields

$$
\left\{\sum_{\alpha \in \mathbb{Z}}\left(\sum_{n=-\infty}^{\alpha} A_{n}\right)^{q}\left\|u \phi_{1} \chi_{\alpha}\right\|_{X}^{q}\right\}^{1 / q} \leq C\left(\sum_{n \in \mathbb{Z}} A_{n}^{b} V_{n}\right)^{1 / b}
$$

where the RHS of the above inequality is dominated by

$$
C\left(\varepsilon^{1-r^{\prime}}\right)^{1 / r}\left(\sum_{n \in \mathbb{Z}} a_{n-1}^{b}\right)^{1 / b}<\infty
$$

since $\left\{a_{n}\right\} \in \ell^{b}$. The inequality (2.11) holds implies by [3, Corollary 1.3],

$$
\tilde{C}_{3}=\sup _{m \in \mathbb{Z}}\left(\sum_{n=m}^{\infty}\left\|u \phi_{1} \chi_{n}\right\|_{X}^{q}\right)^{1 / q}\left\{\sum_{n=-\infty}^{m} V_{n}^{1-b^{\prime}}\right\}^{1 / b^{\prime}}<\infty \text { holds for } b \leq q .
$$

In inequality (2.11), $\mathrm{C}$ is independent of $\varepsilon$. Since $C \sim \tilde{C}_{3}$ for $b \leq q$, we have, by taking $\varepsilon \rightarrow 0$ (via a subsequence) $\tilde{C}_{3}=C_{3}$ and therefore the necessity of $C_{3}<\infty$ for $b \leq q$ follows. Next, we replace $\tilde{v}_{\varepsilon}$ and $\tilde{f}$ defined as in (2.8) and (2.10) by, respectively,

$$
\begin{aligned}
\tilde{v}_{\varepsilon}(x) & =\max \left\{v(x),\left|\psi_{2}(x)\right|^{r} \varepsilon\right\} \text { and } \\
\tilde{f}(x) & =\left\{\begin{array}{cc}
\sum_{n \in \mathbb{Z}} a_{n}\left(\frac{v_{\varepsilon}(x)}{\left|\psi_{2}(x)\right|}\right)^{1-r} \operatorname{sgn}\left(\psi_{2}(x)\right) \chi_{[n, n+1]}(x), & x \geq \alpha \\
0, & x<\alpha
\end{array}\right.
\end{aligned}
$$

where $\alpha \in \mathbb{Z}$ is fixed. The necessity of $C_{4}<\infty$, now, can be achieved by making parallel argument in the inequality (2.9) for this new $\tilde{v}_{\varepsilon}$ and $\tilde{f}$ and using Proposition 1.1.

\section{References}

[1] C. Bennett and R. Sharpley, Interpolation of Operators, Academic Press, 1988. 
[2] E.I. Berezhnoi, Sharp estimates of operators on the cone of ideal spaces, Proc. Steklov Inst. Math. 3 (1994), 3-34.

[3] C. Carton-Lebrun, H.P. Heinig and S.C. Hofmann, Integral operators on weighted amalgams, Studia Math. 109(2) (1994), 133-157.

[4] J.J.F. Fournier and J. Stewart, Amalgams of $L^{p}$ and $\ell^{q}$, Bull. Amer. Math. Soc. 13 (1985), 1-21.

[5] F. Holland, Harmonic analysis on amalgam of $L^{p}$ and $\ell^{q}$, J. London Math. Soc. 10(2) (1975), 295-305.

[6] P. Jain and S. Kumar, Boundedness of Hardy operators on generalized amalgams, Math. Inequal. Appl. 12(3), (2009), 549-562.

[7] P. Jain and S. Kumar, Weighted inequalities of Hardy-type on amalgams, Real Anal. Exchange 34(2) (2008/2009), 483-500.

[8] A. Kufner and L.-E. Persson, Weighted Inequalities of Hardy-Type, World Scientific, New Jersey/London/Singapore/Hong Kong, 2003.

[9] S. Kumar, A Hardy-type inequality in two dimensions, Indag. Math. 20(2) (2009), 247260.

[10] S. Kumar, Hardy inequality in Banach function space, IJPAM, 85(4), 2013, 629-633.

[11] E. Lomakina and V.D. Stepanov, On the Hardy type integral operators in Banach function spaces, Publ. Math. 42 (1998), 165-194.

[12] W.A.J. Luxemburg, Banach Function Spaces, Ph.D. Thesis, Delft Institute of Technology, Aseen (Netherlands), 1955.

[13] N. Wiener, On the representation of functions by trigonometrical integrals, Math. Z. 24 (1926) 575-616.

[14] N. Wiener, Tauberian theorems, Ann. of Math. 33 (1932), 1-100.

[15] P.A. Zharov, On a two-weight inequality, Generalization of inequalities of Hardy and Poincar (Russian), Trudy Mat. Inst. Steklov 194 (1992), 97-110; translation in Proc. Steklov Inst. Math. 194(4) (1993), 101-114. 